\title{
Acquired inhibition of microRNA-124 protects against spinal cord ischemia-reperfusion injury partially through a mitophagy-dependent pathway
}

\author{
Kun Liu, MD, ${ }^{\text {a }}$ Lihui Yan, MD, ${ }^{\text {b,c }}$ Xiaojing Jiang, MD, PhD,${ }^{\mathrm{c}}$ Yang Yu, MD, PhD, ${ }^{\text {a }}$ Hongbo Liu, MD, PhD,
} Tianxiang $\mathrm{Gu}, \mathrm{MD}, \mathrm{PhD},{ }^{\mathrm{a}}$ and Enyi Shi, $\mathrm{MD}, \mathrm{PhD}^{\mathrm{a}}$

\section{ABSTRACT}

Objective: Mitophagy results in selective clearance of damaged mitochondria. We investigated whether mitophagy was involved in the neuroprotection by inhibiting microRNA (miRNA)-124 on ischemic spinal cords.

\begin{abstract}
Methods: Inhibition of miRNA-124 was conducted by intrathecal injection of lentivirus vectors containing antagomiR-124. Spinal cord ischemia was induced in rats by crossclamping the descending aorta just distal to the left subclavian artery for 14 minutes. Hind-limb motor function was assessed with the motor deficit index (MDI). Lumbar spinal cords were harvested for ultrastructural, histologic examinations, and terminal deoxynucleotidyl transferase-mediated dUTP-biotin nick-end labeling staining. Mitophagy was evaluated by expressions of beclin-1 and LC3-II in mitochondria. Expressions of inhibitory member of the apoptosis-stimulating proteins of p53 family, p53, beclin-1, LC3-II, and miRNA-124 were measured by Western blot and quantitative real-time polymerase chain reaction. Mitophagy was inhibited by the antagonist of 3methyladenine.
\end{abstract}

Results: Compared with control animals, antagomiR-124 significantly inhibited expressions of miRNA-124 $(P<.01)$ and $\mathrm{p} 53(P<.05)$ and enhanced expressions of inhibitory member of the apoptosis-stimulating proteins of $\mathrm{p} 53$ family, becline1 and LC3-II $(P<.01$, respectively) in spinal cords. MDI at $6,12,24$, and 48 hours after reperfusion were markedly lower in antagomiR-124 group $(P<.01$, vs control group, respectively). More motor neurons and less apoptotic cells were detected in lumbar spinal cords of antagomiR-124 group $(P<.01$ vs control group). Administration of 3-methyladenine completely abolished enhancements of mitochondrial becline-1 and LC3-II by antagomiR-124 $(P<.01$ vs antagomiR-124 group) and partially inhibited effects of antagomiR-124 on MDI, number of motor neurons, and apoptotic cells $(P<.01$ or $<.05$ vs control group and antagomiR-124 group, respectively).

Conclusions: Inhibition of miRNA-124 exerts neuroprotection on spinal cords against ischemia-reperfusion injury, possibly by induction of mitophagy and antiapoptotic effects. (J Thorac Cardiovasc Surg 2017;154:1498-508)

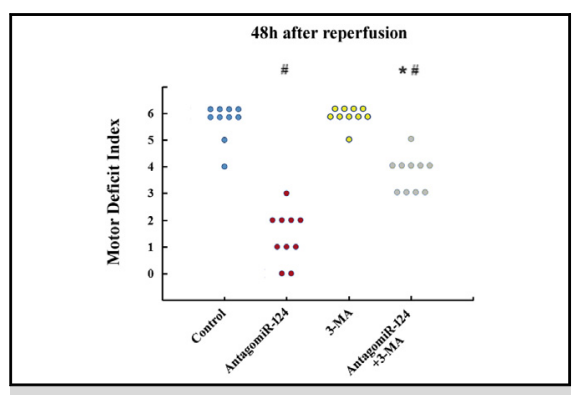

Neuroprotection that results from down-regulation of miR-124 is partially blocked by inhibiting mitophagy. ${ }^{*} P<.01$ compared with the antagomiR-124 group $\# P<.01$ compared with the control group.

\section{Central Message}

Inhibition of microRNA-124 results in neuroprotection against spinal cord ischemiareperfusion injury, which is possibly mediated by induction of mitophagy and antiapoptotic effects.

\section{Perspective}

Inhibition of microRNA (miRNA)-124 provides a powerful neuroprotection on ischemic spinal cords, which is possibly mediated by both induction of mitophagy and antiapoptotic effects. To our knowledge, this is the first report that mitophagy serves as a novel mechanism of neuroprotection on ischemic spinal cords by targeting miRNAs. miRNAs and mitophagy may become potential therapeutic targets for ischemic spinal cords.

See Editorial Commentary page 1509.
Transient or permanent spinal cord ischemia remains the most devastating complication after open and endovascular thoracoabdominal aortic aneurysm repair,

From the Departments of ${ }^{\mathrm{a} C a r d i a c}$ Surgery and ${ }^{\mathrm{c}}$ Anesthesiology, First Affiliated Hospital, China Medical University; ${ }^{\mathrm{b}}$ Department of Anesthesiology, Liaoning Cancer Hospital and Institute; and ${ }^{\mathrm{d} D e p a r t m e n t}$ of Health Statistics, School of Public Health, China Medical University, Shenyang, People's Republic of China.

This work was supported by grant No. 81471267 from the National Natural Science Foundation of China, Beijing, P. R. China.

E.S. and T.G. contributed equally to this work. which is associated with a high incidence of paraplegia. Regardless of the refinements of surgical techniques and perioperative strategies, spinal cord ischemia is still

Received for publication Jan 28, 2017; revisions received April 24, 2017; accepted for publication May 14, 2017; available ahead of print June 13, 2017.

Address for reprints: Enyi Shi, MD, PhD, Department of Cardiac Surgery, First Affiliated Hospital, China Medical University, Shenyang 110001, People's Republic of China (E-mail: shienyi2002@hotmail.com).

0022-5223/\$36.00

Copyright (c) 2017 by The American Association for Thoracic Surgery

http://dx.doi.org/10.1016/j.jtcvs.2017.05.046 


\section{Abbreviations and Acronyms \\ 3-MA $=3$-methyladenine \\ ASPP $=$ apoptosis-stimulating proteins of $\mathrm{p} 53$ \\ iASPP = inhibitory member of the apoptosis- \\ stimulating proteins of $\mathrm{p} 53$ family \\ MDI $=$ Motor deficit index \\ miRNA $=$ microRNA \\ ROS $=$ reactive oxygen species \\ $\mathrm{TU}=$ transfection units \\ TUNEL $=$ terminal deoxynucleotidyl transferase- mediated dUTP-biotin nick-end labeling}

Scanning this QR code will take you to the article title page.

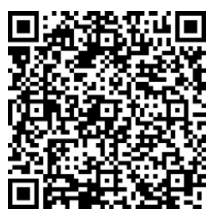

understood incompletely and not entirely predictable or preventable. ${ }^{1,2}$

microRNAs (miRNAs) are 21- to 23-nucleotide nonprotein-coding RNA molecules that act as negative regulators of gene expression by either degrading the target mRNAs or arresting their translation. ${ }^{3}$ miRNAs have been shown to be abundant in central nervous system and play an important role in the development of neurons, maintenance of the neuron phenotype, neurodegeneration, and neuroprotection against ischemia and injury. ${ }^{4,5}$ Transient focal ischemia induces extensive temporal changes in rat cerebral microRNAome. ${ }^{6}$ Expressions of a large set of miRNAs targeting components that are involved in the inflammation, oxidation, and apoptosis are altered after a spinal cord contusive injury. ${ }^{7}$ Inhibition of miRNA-29c has been shown to protect the brain in a rat model of prolonged hypothermic circulatory arrest. ${ }^{8}$ In our previous reports, inhibition of miRNA-320 and overexpression of miRNA-21 are indicated to induce neuroprotection on rat spinal cords against transient ischemia. ${ }^{9,10}$ Collectively, available findings suggest that miRNAs may be an attractive therapeutic target for spinal cord ischemia-reperfusion injury.

Mitochondria serve as the powerhouse of cells, respond to cellular demands and stressors, and play an essential role in cell signaling, differentiation, and survival. In response to changes in the intracellular environment, mitochondria become producers of excessive reactive oxygen species (ROS) and release prodeath proteins, resulting in disrupted ATP synthesis and activation of cell death pathways. ${ }^{11}$ Autophagy is a cellular housekeeping function responsible for the bulk degradation of large protein aggregates or damaged organelles. ${ }^{12}$ Mitophagy, the mitochondrial autophagy, results in selective clearance of damaged mitochondria in cells before they cause activation of cell death. ${ }^{11}$ Therefore, mitophagy is a crucial mechanism to control the quality of mitochondria by preventing the generation of ROS from dysfunctional mitochondria. ${ }^{13}$ miRNAs can affect the mitochondrial metabolism, participate in the regulation of mitochondria-mediated apoptosis, and regulate mitochondrial morphology. ${ }^{14}$ Furthermore, recent works have suggested a potential role of miRNAs in controlling autophagy, including miRNA-101, ${ }^{15}$ miRNA-204, ${ }^{16}$ and miRNA-30a. ${ }^{17}$ However, it remains unknown whether miRNAs regulate mitophagy in the pathologic process of spinal cord ischemia-reperfusion injury.

miRNA-124 is one of the most abundant miRNAs in adult and embryonic brain ${ }^{18}$ and is involved in the transformation from the neuronal stem cells to mature neurons. ${ }^{19}$ Plasma miRNA-124 has been found to be increased in rats after cerebral ischemia, suggesting its potential role as a biomarker for cerebral infarction. ${ }^{20}$ Knockdown or inhibition of cerebral miRNA-124 reduces cell death and infarct size and improves neurologic outcomes. ${ }^{21}$ In the current study, we highlighted the possible neuroprotective effects of inhibition of miRNA-124 on spinal cords against transient ischemia and tried to explore the role of mitophagy in such neuroprotection. Inhibitory member of the apoptosisstimulating proteins of p53 family (iASPP) was indicated to be a target protein of miRNA-124 with the help of a bioinformatics-based database (http://www.targetscan. org $)^{22}$ and published reports. ${ }^{23}$ After inhibition of miRNA124 and transient spinal cord ischemia, expression of iASPP and its downstream protein p53 were measured. To evaluate the role of mitophagy in spinal cords suffering from transient ischemia, mitochondrial expressions of beclin-1 and LC3-II (molecular markers of autophagy) were measured.

\section{METHODS}

\section{Animals}

Male Wistar rats weighing about $250 \mathrm{~g}$ were enrolled in the present study. The animal protocol was approved by the Ethics Review Committee for Animal Experimentation of China Medical University (Shenyang, People's Republic of China). It was conducted in accordance with the Guide for the Use and Care of Laboratory Animals (National Institutes of Health, Bethesda, Md).

\section{Inhibition of miRNA-124 In Vivo}

Chemically modified antisense oligonucleotides of rat miRNA-124 (antagomiR-124) lentivirus gene transfer vectors were constructed by Genechem (Shanghai, China). Lentivirus vectors without antagomiR-124 were used as control vectors. The lentivirus vector of antagomiR-124 and control vector were prepared and titered to $1 \times 10^{9}$ transfection units (TUs) $\cdot \mathrm{mL}^{-1}$, according to manufacturer guidelines.

Lentivirus vectors of antagomiR-124 or control vectors were transfected in vivo by means of intrathecal injection, as reported previously. 9,10 Animals were included in the following protocol only if they had normal hind-limb motor function 3 days after the intrathecal injection. 
Part I

Sham

Control

Control vector

antagomiR-124

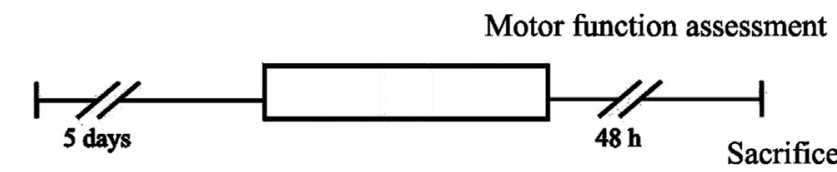

vehicle $10 \mu$

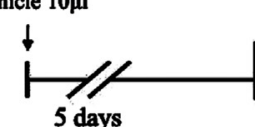

aortic cross-clamping $14 \mathrm{~min}$

Motor function assessment

$48 \mathrm{~h}$

Sacrifice

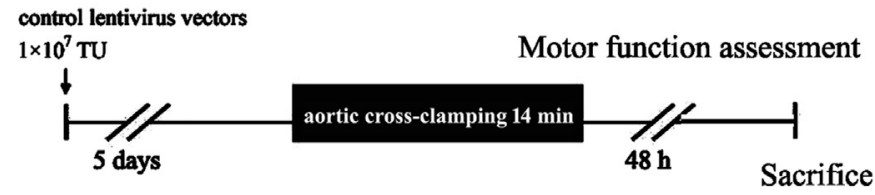

lentivirus of antagomiR- 124 $1 \times 10^{7} \mathrm{TU}$

Motor function assessment

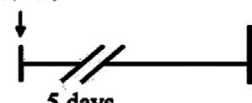

aortic cross-clamping $14 \mathrm{~min}$

Sacrifice

\section{Part II}

Control

antagomiR-124

3-MA

antagomiR-124 +3-MA

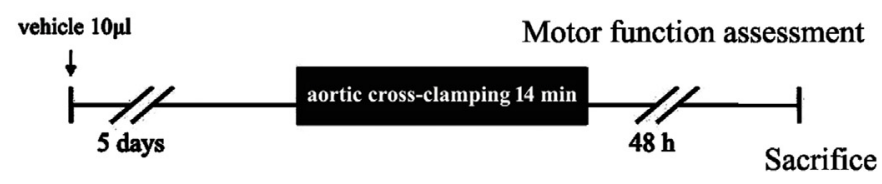

lentivirus of antagomiR- 124 $1 \times 10^{7} \mathrm{TU}$

Motor function assessment

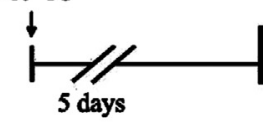

aortic cross-clamping $14 \mathrm{~min}$

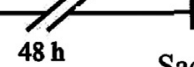

Sacrifice
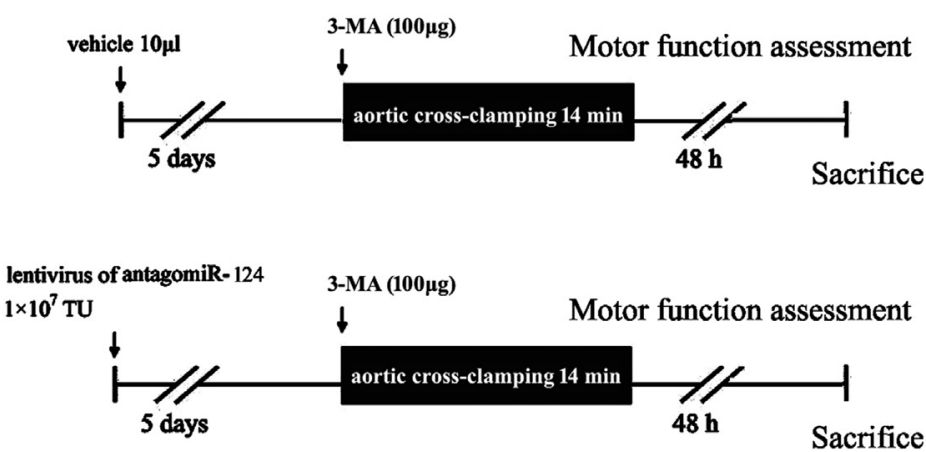

FIGURE 1. Experimental groups and protocol. 3-MA, 3-Methyladenine.

\section{Spinal Cord Ischemia}

Spinal cord ischemia was induced according to the method described previously. ${ }^{9,10}$ In summary, core body temperature was maintained at $37 \pm 0.5^{\circ} \mathrm{C}$ with the aid of a heat lamp. A tracheotomy was performed, and the animal was ventilated mechanically. A 24-gauge catheter was inserted into the tail artery to measure distal blood pressure. Another 24-gauge catheter was inserted into the left carotid artery to measure proximal blood pressure and also was connected to an external blood reservoir positioned at a height of $54 \mathrm{~cm}$ above the level of the rat's body to maintain the mean proximal blood pressure at about $40 \mathrm{~mm} \mathrm{Hg}$ during aortic 
occlusion. Through a left thoracotomy, the descending aorta was crossclamped just distal to the left subclavian artery for 14 minutes after systemic heparinization $(200 \mathrm{U} / \mathrm{kg})$. The ischemia was confirmed by a reduction of distal blood pressure to $<10 \mathrm{~mm} \mathrm{Hg}$. At the end of the procedure, the clamp was removed and the blood in the external blood reservoir was reinfused.

\section{Experimental Protocol}

Experimental groups and protocol are shown in Figure 1.

Part I. To evaluate the neuroprotection of inhibition of miRNA-124, 4 groups were enrolled. (1) Sham group $(\mathrm{n}=10)$ : rats received only the surgical procedure without spinal cord ischemia. (2) Control group $(\mathrm{n}=11)$ : vehicle $(10 \mu \mathrm{L})$ were injected intrathecally into each rat 5 days before a 14-minute spinal cord ischemia. (3) Control vector group $(\mathrm{n}=12)$ : approximately $1 \times 10^{7} \mathrm{TU}$ control lentivirus vectors $(10 \mu \mathrm{L})$ were injected intrathecally into each rat 5 days before a 14-minute spinal cord ischemia. (4) AntagomiR-124 group $(\mathrm{n}=10)$ : approximately $1 \times 10^{7} \mathrm{TU}$ lentivirus vectors of antagomiR-124 (10 $\mu \mathrm{L})$ were injected intrathecally into each rat 5 days before a 14-minute spinal cord ischemia. In a parallel series of experiments, spinal cords were additionally collected 6 hours after reperfusion from the 4 groups ( $\mathrm{n}=4$ per group), to evaluate expressions of iASPP, p53, beclin-1, LC3 II, and miRNA-124.

Part II. Another 4 groups were enrolled to evaluate the role of mitophagy using the selective inhibitor of mitophagy 3-methyladenine (3-MA; Sigma Chemical, St Louis, Mo). The protocols of (1) control group $(\mathrm{n}=11)$ and (2) antagomiR-124 group $(\mathrm{n}=11)$ were the same as Part I. 3-MA (100 $\mu \mathrm{g}, 2 \mu \mathrm{L}$ in saline) was administrated by means of intrathecal injection before spinal cord ischemia to (3) 3-MA group $(\mathrm{n}=10)$ and (4) antagomiR$124+3$-MA group $(n=12)$. In a parallel series of experiments, spinal cords additionally were collected 6 hours after reperfusion from the 4 groups $(n=4$ per group), to evaluate expressions of beclin-1, LC3 II, and miRNA-124.

\section{Neurologic Assessment}

Hind-limb motor function was assessed at 6, 12, 24, and 48 hours after reperfusion, via the motor deficit index (MDI) score (quantified by ambulation and the placing/stepping reflex $)^{24}$ by investigators who were blinded to group information. Hind-limb ambulation was graded as follows: 0, normal (symmetric and coordinated ambulation); 1, toes flat under the body when walking, but ataxia present; 2 , knuckle walking; 3 , unable to knuckle walk but some movement of the hind limbs; and 4, no movement, or drags lower extremities. The placing/stepping reflex was assessed by the dragging movements and responses of the hind-paw dorsum when touching the floor surface. A coordinated lifting and placing response, which generally was evoked when a hind paw touched the ground, was graded as follows: 0 , normal; 1 , weak; and 2, no stepping. The MDI score was calculated for each rat as the sum of these scores at each interval.

\section{miRNA Extraction and Quantitative Real-Time Polymerase Chain Reaction}

Total RNA was isolated by with Trizol reagent (Invitrogen, Carlsbad, Calif) according to the manufacturer's instruction. The first-strand complementary DNA was generated with the Reverse Transcription System Kit (Invitrogen). Real-time polymerase chain reaction was performed according to a standard protocol with the StepOne Plus system (Applied Biosystems, Foster City, Calif). U6 served as an internal control. Changes in expression were determined by the $2^{-\Delta \Delta \mathrm{CT}}$ method.

\section{Western Blot}

Mitochondrial protein was extracted with an isolation kit (Beyotime, Shanghai, China) according to the protocol recommended by the manufacturer. Mitophagy was evaluated by expressions of beclin-1 and LC3-II in the mitochondrial protein. iASPP and p53 in spinal cords and mitochondrial beclin-1 and LC3-II were analyzed by sodium dodecyl sulfate polyacrylamide gel electrophoresis and immunoblotted with anti-iASPP, anti-p53, anti-beclin-1, and anti-LC3 antibodies (Abcam, Cambridge, Mass). The protein expressions in each spinal cord tissue sample were analyzed with NIH Image (Research Services Branch, National Institutes of Health) and quantified as a relative fold to the sham group or the control group after normalization with glyceraldehyde 3-phosphate dehydrogenase.

\section{Histologic Study}

For histologic study, rats were killed 48 hours after reperfusion. Paraffin-embedded sections ( $4 \mu \mathrm{m})$ of lumbar spinal cords (L4-L6) were stained with hematoxylin-eosin and Nissl dye. Gray-matter damage was assessed by counting the number of normal motor neurons in the ventral part of the gray matter (anterior to a transverse line drawn through the central canal), at the original magnification, $\times 400$. Cells that contained Nissl substance in the cytoplasm, loose chromatin, and prominent nucleoli were considered normal motor neurons. The number of normal motor neurons in each animal was obtained by averaging counts from 3 different slides.

\section{Transmission Electron Microscopic Examination}

The tissue samples were handled as reported previously. ${ }^{25}$ To summarize, spinal cord section was quickly cut into 1-mm cubes, immersionfixed with $2.5 \%$ glutaraldehyde in $0.1 \mathrm{~mol} / \mathrm{L}$ phosphate buffer $(\mathrm{pH} 7.4)$ overnight at $4{ }^{\circ} \mathrm{C}$, and fixed in $1 \%$ buffered osmium tetroxide. Specimens were dehydrated through a graded ethanol series and embedded in epoxy resin.

\section{Terminal Deoxynucleotidyl Transferase-Mediated dUTP-Biotin Nick-End Labeling (TUNEL) Staining}

Frozen sections were used for in situ TUNEL staining using a one-step TUNEL labeling kit (Beyotime, Shanghai, China). Fluorescence signals were visualized via fluorescence microscopy and counted in the ventral part of the gray matter.

\section{Statistical Analysis}

Parametric values (expressions of miRNA-124, iASPP, p53, beclin-1, LC3-II) are reported as mean \pm standard deviation. Differences were analyzed by one-way analysis of variance followed by Bonferroni correction for post-hoc testing. MDI scores, numbers of neurons, and apoptotic cells were analyzed by Kruskal-Wallis test with Bonferroni correction $(P<.008$ before Bonferroni correction was considered statistically significant). Statistical significance was defined as $P<.05$. Statistical analysis was performed with SPSS, version 19.0 (IBM-SPSS Inc, Armonk, NY).

\section{RESULTS \\ Expressions of miRNA-124, iASPP, and p53 After Transfection of AntagomiR-124}

Results of quantitative real-time polymerase chain reaction are summarized in Figure 2, A. The expression of miRNA-124 could be detected in spinal cords of sham rats and decreased slightly in the control group after reperfusion ( $P=1.000$, vs the sham group). Transfection of antagomiR-124 significantly decreased the expression of miRNA-124 in spinal cords after reperfusion $(P=.003$, vs the control group). Control vector did not affect the expression of miRNA-124 $(P=1.000$, vs the control group).

Representative western blot pictures of iASPP and p53 expressions are shown in Figure 2, B. Densitometric analysis revealed that transfection of antagomiR-124 markedly 

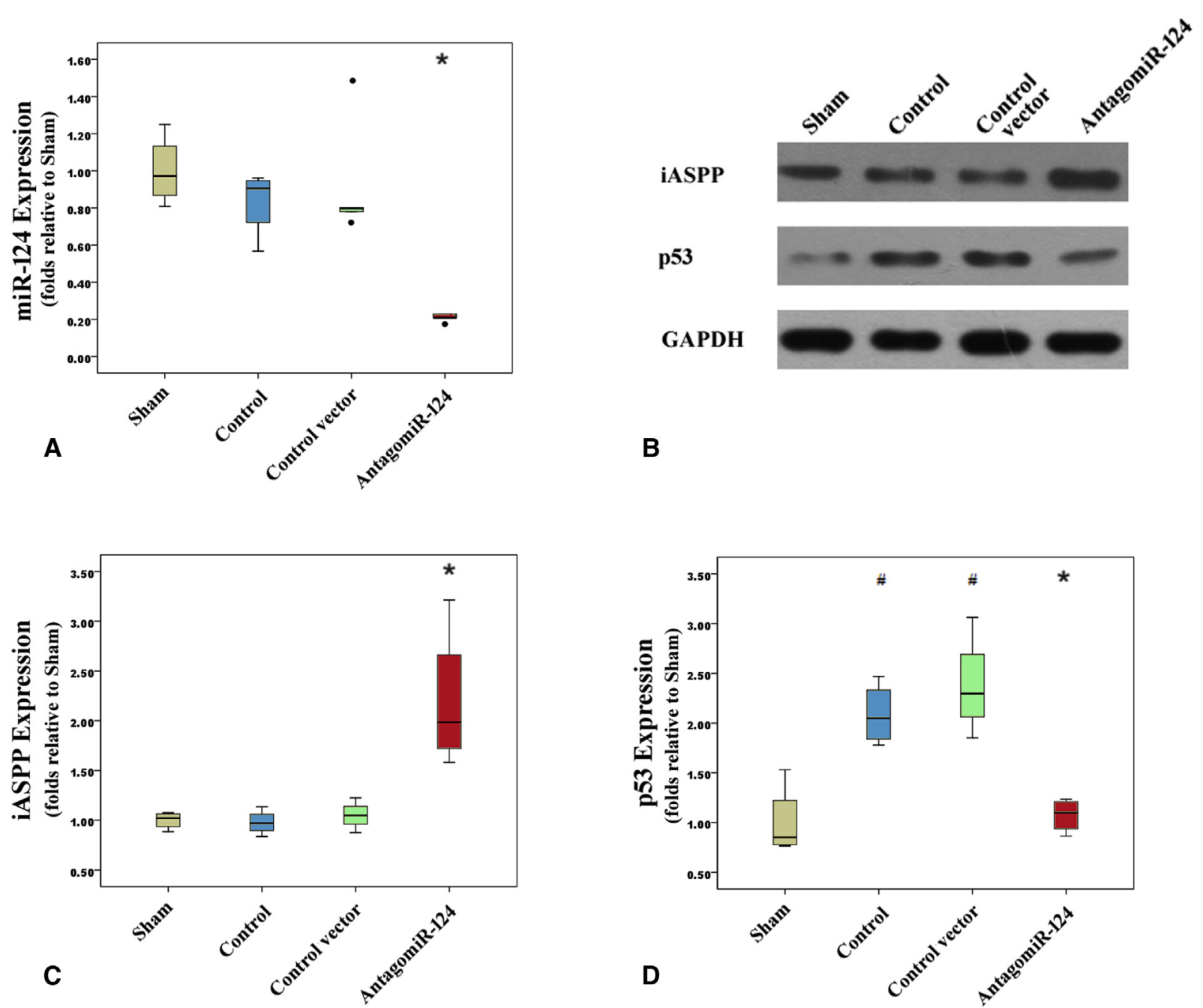

FIGURE 2. Effects of antagomiR-124 administration on expressions of miRNA-124, iASPP and p53 in lumber spinal cords 6 hours after reperfusion. A, miRNA-124 expression. B, Representative Western blot pictures showing iASPP and p53 expressions. C, Densitometric quantification of iASPP expression. $\mathrm{D}$, Densitometric quantification of 533 expression. ${ }^{*} P<.05$, compared with the control group; $\# P<.01$, compared with the sham group. $i A S P P$, Inhibitory member of the apoptosis-stimulating proteins of p53 family; GAPDH, glyceraldehyde-3-phosphate dehydrogenase.

increased the expression of iASPP in spinal cords $(P=.004$, vs the control group, Figure 2,C). There was no significant difference in the expression of iASPP between the control group and the control vector group $(P=1.000$, Figure $2, C)$.

After reperfusion, the expression of p53 was enhanced dramatically in spinal cords of the control group and the control vector group $(P=.007$ and $P=.001$, vs the sham group respectively, Figure 2, $D$ ), whereas such an enhancement of p53 expression was inhibited completely after transfection of antagomiR-124 $(P=.011$, vs the control group; $P=1.000$, vs the sham group, Figure $2, D$ ).

\section{Effect of AntagomiR-124 on the Induction of Mitophagy}

Mitochondrial beclin-1 and LC3-II were measured as molecular markers of mitophagy. As illustrated in Figure 3, $A$ and $B$, the expression of beclin-1 in rats pretreated with antagomiR-124 was much higher than that in the control group $(P<.001)$. Compared with the sham group, slight increases of the expression of beclin-1 in the control group and the control vector group were detected after reperfusion, but the differences did not reach a statistical significance $(P=.599$ and $P=.860$, respectively).

Densitometric analysis revealed that the expression of LC3-II also was enhanced markedly by pretreatment of antagomiR-124 ( $P=.003$, vs the control group, Figure 3, $A$ and $C$ ). Compared with the sham group, no significant difference in LC3-II expression was detected in either the control group $(P=.274)$ or the control vector group $(P=.248)$.

\section{Transfection of AntagomiR-124 Improves Neurologic Function}

A total of 43 rats were enrolled in the protocol of part I to evaluate neuroprotection of inhibition of miRNA-124. 


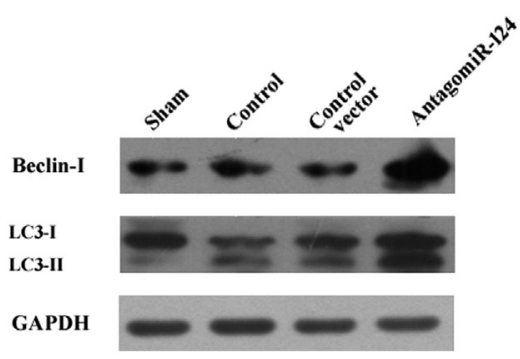

A

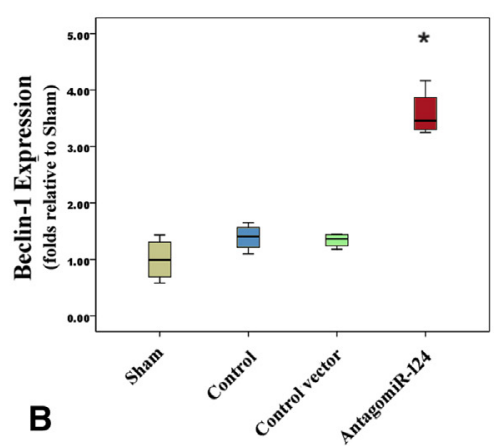

FIGURE 3. Effects of antagomiR-124 administration on expressions of beclin-1 and LC3 II in lumber spinal cords 6 hours after reperfusion. A, Representative Western blot pictures showing beclin-1 and LC3 II expressions. B, Densitometric quantification of beclin-1 expression. C, Densitometric quantification of LC3 II expression. $* P<.01$ compared with the control group. GAPDH, Glyceraldehyde-3-phosphate dehydrogenase.

Three rats were excluded because of death during the operation or not surviving for 48 hours after the transient spinal cord ischemia. Complete data were obtained in the remaining 40 rats ( $n=10$ for each group). Figure 4 summarizes the individual MDI scores of the 4 groups in experiment Part I. Spinal cord ischemia for 14 minutes induced severe neurologic injury in both the control group and the control vector group at the 4 observation points after reperfusion $(P<.001$, vs the sham group, respectively). Pretreatment of antagomiR-124 significantly decreased the MDI scores at the 4 observation time points after reperfusion $(P<.001$, vs the control group, respectively).
Effects of AntagomiR-124 on Apoptosis, Ultrastructural, and Histologic Examinations

A normal mitochondrion in a motor neuron of the sham group is shown in Figure 5, A- $a$. A typical mitophagy in a rat of the antagomiR-124 group was verified by the mitochondria encapsulated by the double membrane of an autophagosome, as illustrated in Figure 5, A- $b$.

Representative sections of lumbar spinal cords stained with HE and Nissl dye are shown in Figure 5, $A-c, d, e$, and $f$, and the results of counting normal motor neurons are summarized in Figure 5, $B$. Severe neurologic damage of spinal cords characterized by vacuolization, frank
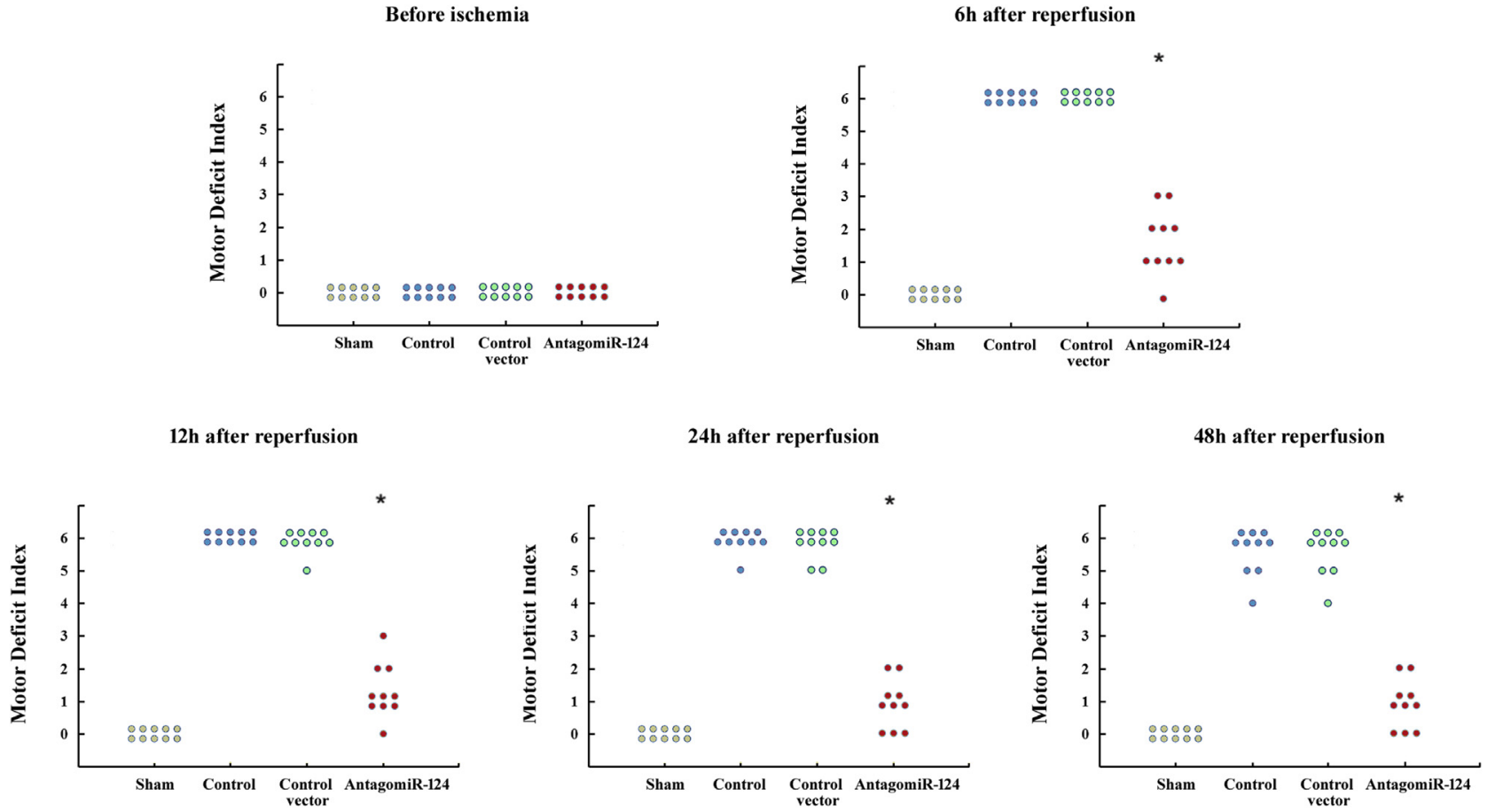

FIGURE 4. Effects of antagomiR-124 pretreatment on hind-limb motor function assessed with the motor deficit index before spinal cord ischemia, and 6 , 12,24 , and 48 hours after reperfusion. $* P<.01$ compared with the control group. 

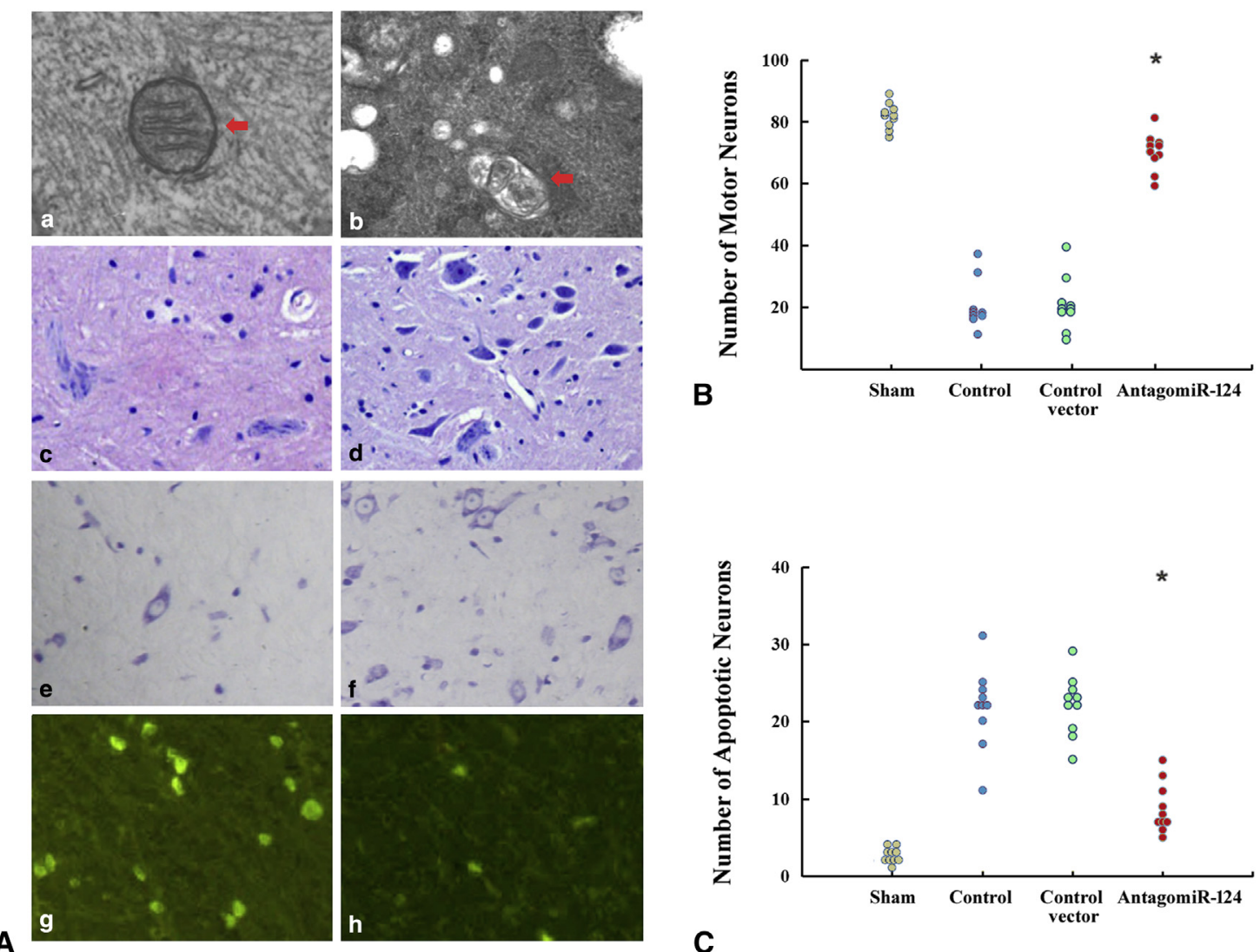

FIGURE 5. Ultrastructural and histological examinations of lumber spinal cords. A, Representative electron micrographs and HE, Nissl, and TUNEL staining sections of spinal cords. $a$, The ultrastructure of a normal mitochondrion in the motor neuron of the sham group (arrow). $b$, Mitochondria within autophagosome-like structures in the motor neuron of the antagomiR-124 group (arrow). $c$ and $d$, HE staining sections of the control group (c) and the antagomiR-124 group $(d) . e$ and $f$, Nissl staining sections of the control group $(e)$ and the antagomiR-124 group $(f)$. $g$ and $h$, TUNEL staining sections of the control group $(g)$ and the antagomiR-124 group $(h)$. Green represents TUNEL-positive cells. B, Number of motor neurons in the ventral gray matter of lumber spinal cords. C, Number of apoptotic cells in the ventral gray matter of lumber spinal cords. $* P<.01$ compared with the control group.

necrosis, and loss of motor neurons was detected in both the control group and the control vector group. Slighter histologic changes were found in lumbar spinal cords of animals in the antagomiR-124 group, and the number of intact motor neurons in the antagomiR-124 group was much greater than that in the control group $(P<.001)$.

TUNEL-positive cells represent apoptotic cells in spinal cords as shown in Figure 5, $A-g, h$. Many apoptotic cells were detected in spinal cords of the control group, and the number of apoptotic cells of the control group was much higher than that of the antagomiR-124 group $(P<.001)$.

\section{Effects of 3-MA on the Expression of miRNA-124 and the Induction of Mitophagy}

Administration of 3-MA did not affect the expression of miRNA-124 in spinal cords. No significant difference in miRNA-124 expression was detected between the control group and the 3-MA group $(P=.209$, Figure $6, A)$. Compared with the control group, expression of miRNA124 was dramatically inhibited in both the antagomiR-124 group and the antagomiR-124 + 3-MA group $(P<.001$, respectively).

Western blot of mitochondrial beclin-1 and LC3-II indicated that the induction of mitophagy by antagomiR124 was inhibited markedly by 3-MA administration (Figure 6, B). Compared with the antagomiR-124 group, expressions of both beclin-1 and LC3-II in the antagomiR-124 + 3-MA group were reduced dramatically $(P<.001$ and $P=.007$, Figure $6, C$ and $D)$.

\section{Effects of 3-MA on the Neurologic Function}

Another 44 rats were enrolled in the protocol of part II to evaluate the function of mitophagy in neuroprotection mediated by antagomiR-124. Three rats did not survive for 48 hours after reperfusion. Intrathecal injection was failed in 1 rat. Final data were obtained in the remaining 40 rats $(\mathrm{n}=10$ for each group). As summarized in Figure 7, MDI scores of the antagomiRNA-124 + 3-MA group were much greater than those of the antagomiRNA-124 group $(P<.001)$. However, the MDI scores of the antagomiRNA-124 + 

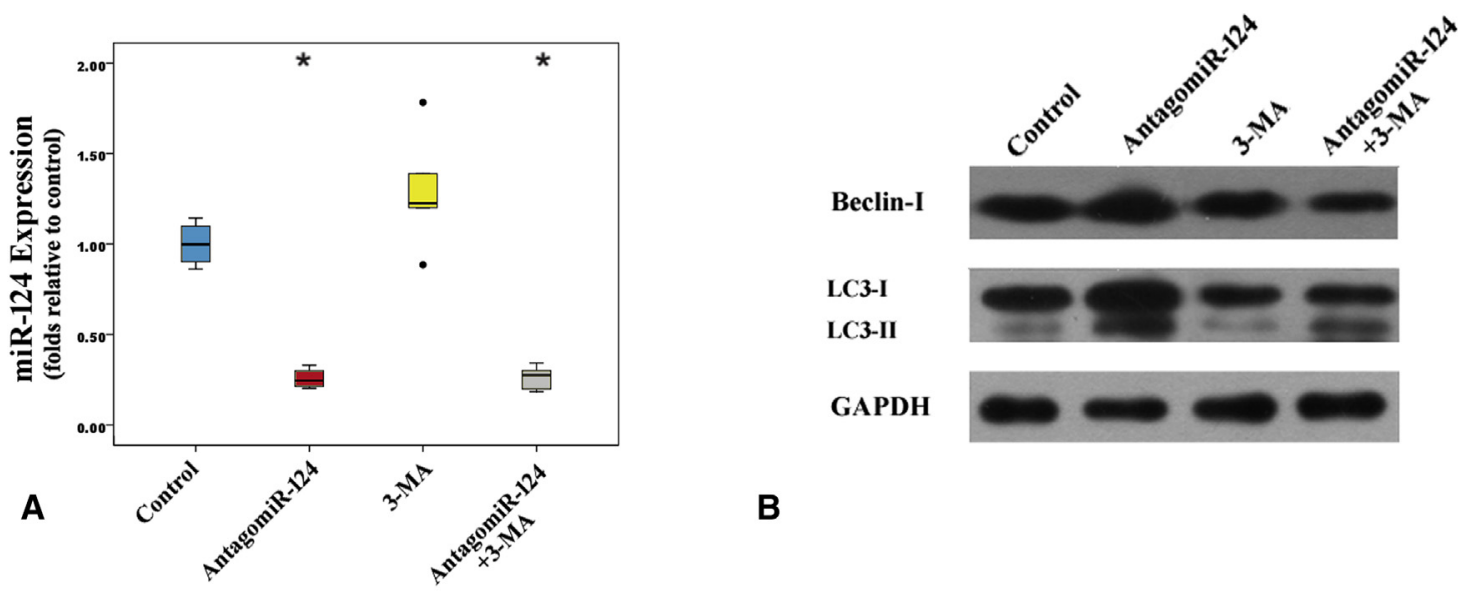

B
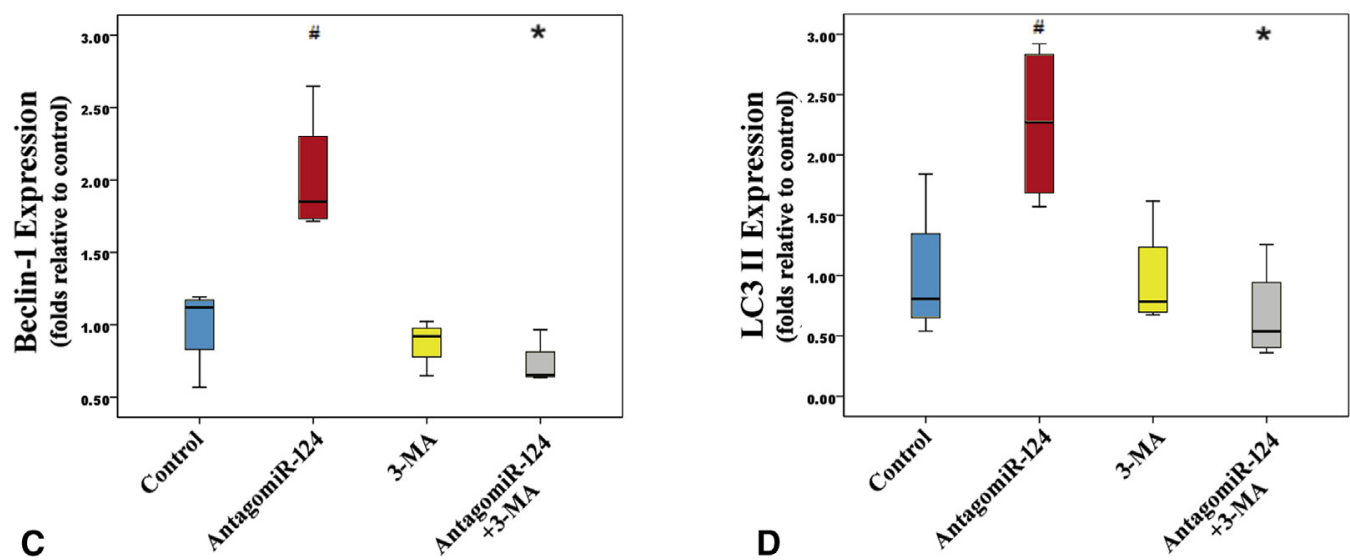

FIGURE 6. Effects of 3-MA on expressions of beclin-1 and LC3 II. A, miRNA-124 expression. B, Representative Western blot pictures showing beclin-1 and LC3 II expressions. C, Densitometric quantification of beclin-1 expression. D, Densitometric quantification of LC3 II expression. *P<.01, compared with the antagomiR-124 group; $\# P<.01$ compared with the control group. 3-MA, 3-Methyladenine; GAPDH, glyceraldehyde-3-phosphate dehydrogenase.

3-MA group were even lower than those of the control group $(P<.001)$.

\section{Effects of 3-MA on the Apoptosis and Histologic Examination}

The number of intact motor neurons in the ventral part of the gray matter of the antagomiR-124 + 3-MA group was much lower than that of the antagomiR-124 group $(P<.001$, Figure 8, $A$ and $C)$. When compared with the control group, much more intact motor neurons were even detected in the antagomiR-124 + 3-MA group $(P=.001)$.

The antiapoptotic effect of antagomiR-124 on ischemic spinal cords was weakened by 3-MA, as evidenced by a higher number of apoptotic cells in the antagomiR-124 + 3-MA group ( $P=.001$, vs the antagomiR-124 group, Figure $8, B$ and $D$ ). However, the number of apoptotic cells in the antagomiR-124 + 3-MA group was still lower than that of the control group $(P=.028)$.

\section{DISCUSSION}

The salient findings revealed by the current study are that inhibition of miRNA-124 in a rat model of spinal cord ischemia is associated with (1) increased expression of iASPP, (2) decreased expression of p53, (3) enhanced mitophagy, (4) improved neurologic function, and (5) fewer apoptotic cells. This effect on neurologic function and apoptosis is partially blocked by an inhibitor of mitophagy, 3-MA.

Collective data have indicated that miRNAs are the novel molecular targets for neuroprotection against ischemia. miRNAs and their target genes are involved in endothelial dysfunction, dysregulation of neurovascular integrity, edema formation, proapoptosis, inflammation, and extracellular matrix remodeling, which are related to the critical processes in the pathogenesis of cerebral ischemia. ${ }^{26}$ miRNA-124 has been shown to be a crucial endogenous regulator in the ischemia-reperfusion-induced brain injury and dysfunction. However, paradoxical results have been 
Before ischemia

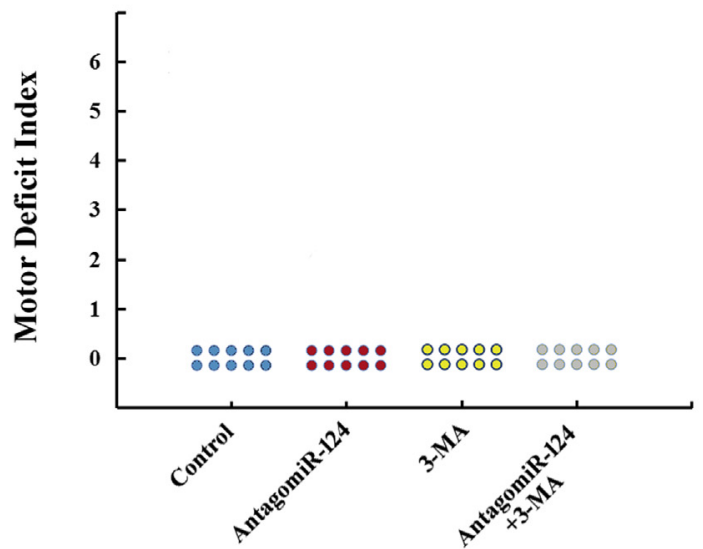

48h after reperfusion

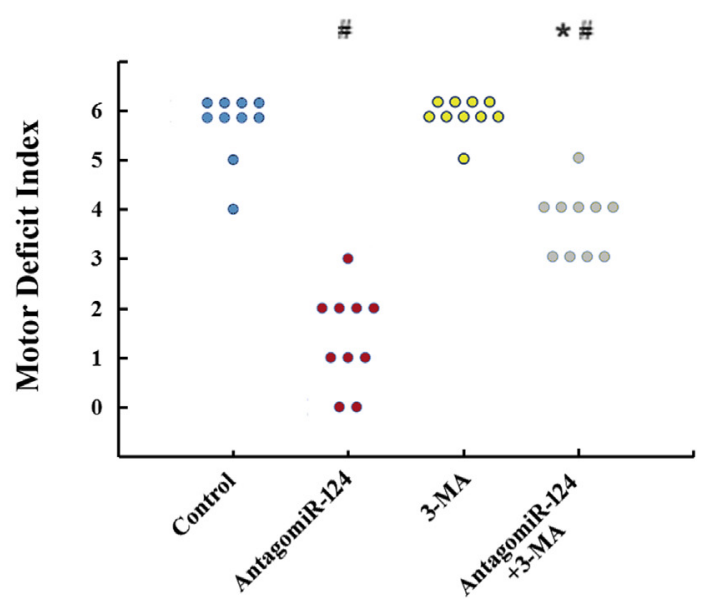

FIGURE 7. Effects of 3-MA on hind-limb motor function assessed using the Motor Deficit Index before spinal cord ischemia and 48 hours after reperfusion. $* P<.01$ compared with the antagomiR-124 group. $\# P<.01$ compared with the control group. 3-MA, 3-Methyladenine.

drawn. miRNA-124 has been suggested to promote neuronal survival under ischemic conditions, ${ }^{27}$ whereas inhibition of miRNA-124 has been shown to induce neuroprotection after experimental stroke in other studies. ${ }^{21,23}$ In the current study, miRNA-124 was confirmed to be expressed in spinal cords, and inhibition of miRNA-124 in ischemic spinal cords was associated with antiapoptotic effects, induction of mitophagy and improved neurological outcomes. Each miRNA has a series of potential target proteins and the functional target protein may be decided by the pathological conditions, degree of the stress, the intervention time point and the effector organ, which may result in the discrepancy of the final outcome of regulation of miRNA-124.

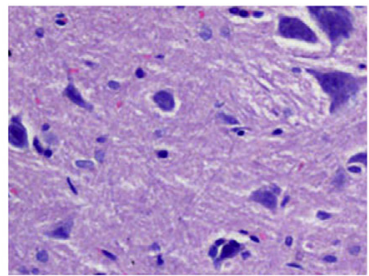

A AntagomiR-124

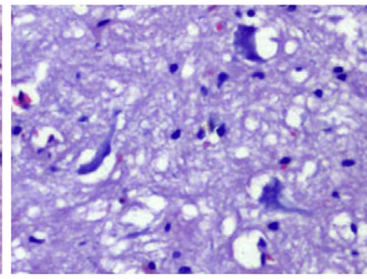

AntagomiR-124+3-MA

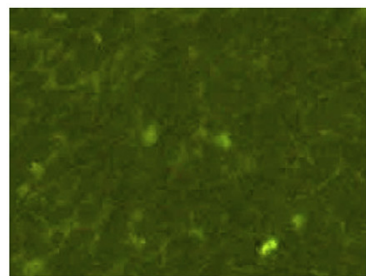

B AntagomiR-124

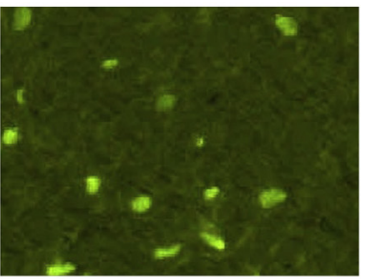

AntagomiR-124+3-MA
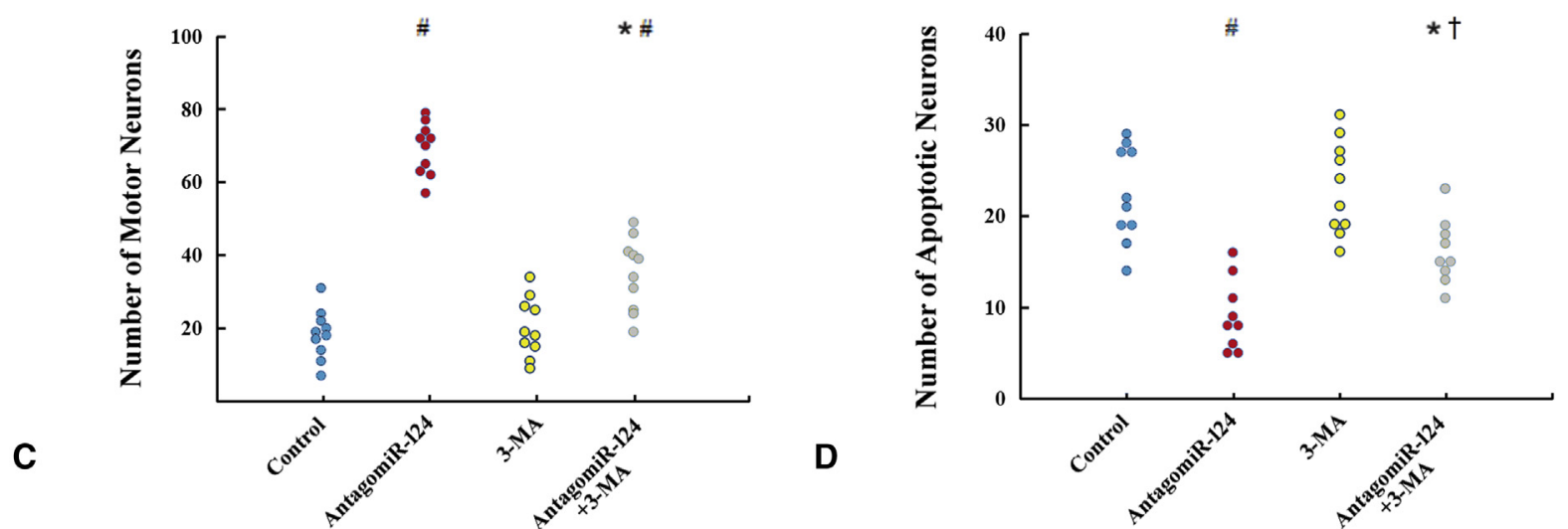

FIGURE 8. Effects of 3-MA on numbers of motor neurons and apoptotic neurons in the ventral gray matter of lumber spinal cords. A, Representative pictures of HE staining sections of lumber spinal cords. B, Representative pictures of TUNEL staining sections of lumber spinal cords. Green represents TUNEL-positive neurons. C, Number of motor neurons. D, Number of apoptotic neurons. $* P<.01$ compared with the antagomiR-124 group; $\# P<.01$, compared with the control group; $\nmid P<.05$ compared with the control group. 3-MA, 3-Methyladenine. 
To elucidate the potential mechanism of the neuroprotection induced by inhibition of miRNA-124 against spinal cords ischemia-reperfusion injury, possibilities for the target protein were explored. miRNA-124 is indicated to be an endogenous regulator of iASPP. The molecular interaction between iASPP and miRNA-124 forms a complex signal transduction pathway to control tumor apoptosis and growth. ${ }^{28}$ miRNA-124 can bind to the $3^{\prime}$-untranslated region of iASPP in $293 \mathrm{~T}$ cells and down-regulate its protein levels in Neuro-2a cells. Furthermore, p53-mediated neuronal cell death after stroke can be nontranscriptionally decreased by an inhibiting-miRNA-124-induced upregulation of iASPP. ${ }^{23}$ p53 plays critical roles in regulating cellular stress by the induction of apoptosis in cerebral ischemia. ${ }^{29}$ p53 participates in the protective effect of ischemic postconditioning against oxygen and glucose deprivation-reperfusion injury in primary cultured spinal cord neurons. ${ }^{30}$ The apoptosis-stimulating proteins of $\mathrm{p} 53$ (ASPP) family comprises a central set of proteins regulating wild-type p53 function in tumors. ASPP family consists of ASPP1 and ASPP2, and functions as tumor suppressors whereas the iASPP functions as oncogene. ${ }^{28}$ In the current study, expression of iASPP was up-regulated by inhibition of miRNA-124 in rat spinal cords. p53 expression in spinal cords was detected to be enhanced after reperfusion and such an overexpression of p53 was inhibited after the expression of iASPP was increased. Hence, it is likely that the iASPP-p53-dependent pathway is involved in the antiapoptotic effects and neuroprotection on ischemic spinal cords conducted by inhibition of miRNA- 124 .

Mitophagy functions as selective clearance of damaged mitochondria to reduce necrosis or apoptosis and to sustain cellular activity. ${ }^{11}$ Evidences indicate that mitophagy is essential for cardioprotection induced by ischemic preconditioning and acute simvastatin treatment. ${ }^{31,32}$ Deficiency in mitophagy is associated with neurodegenerative diseases such as Parkinson, Alzheimer, and Huntington disease. ${ }^{33,34}$ Mitophagy plays important roles in cerebral ischemia and subsequent reperfusion and protects against neuronal injury by mitochondrial clearance. ${ }^{35}$ Mitophagy also is involved in the neuroprotection against acute cerebral ischemic injury by methylene blue and endoplasmic reticulum stress. ${ }^{22,36}$ Mitochondria are the powerhouse of the cell. After cerebral ischemia, mitochondria overproduce ROS which induces cell death through the release of proapoptotic proteins such as cytochrome c or apoptosisinducing factor. Although the mechanisms of cell death after cerebral ischemia remain unclear, mitochondria obviously play a role by activating signaling pathways through ROS production and by regulating mitochondriadependent apoptosis pathways. ${ }^{37}$ Thus, mitophagy is crucial in neuroprotection against ischemia-reperfusion by controlling the quality of mitochondria and preventing the generation of ROS by dysfunctional mitochondria. ${ }^{13}$
Consistently, the mitophagy in spinal cords was enhanced by inhibition of miRNA-124 after reperfusion in the current study, and the improvements of neurologic function and antiapoptotic effects were abolished partially by the antagonist of mitophagy 3-MA, indicating that the mitophagy was involved in mediating the neuroprotection of inhibition of miRNA-124. In contrast, mitophagy excessively activated in myocardial ischemia-reperfusion can promote loss of myocytes. ${ }^{38}$ Suppressing excessive mitophagy also has been shown to mediate neuroprotection of mitochondrial calcium uniporter ${ }^{39}$ and p38 inhibitors. ${ }^{40}$ Mitochondrial dynamics is regulated by both mitochondrial depletion and biogenesis. Excessive depletion of mitochondria by mitophagy may induce an energetic crisis and cellular injuries under ischemia-reperfusion.

Mitophagy is regulated by an increasing number of proteins that reside in the mitochondria or become associated with mitochondria when required, such as PINK1, parkin, BNIP3, NIX, and FUNDC1. The expression and function of some of these proteins are regulated by p53. ${ }^{41}$ Evidences also indicate that p53 attenuates myocyte mitophagy to exacerbate cardiac damage after ischemia. ${ }^{42}$ In the current study, iASPP was highlighted as the target protein of miRNA-124. Hence, it was possible that the induction of mitophagy by inhibition of miRNA-124 was conducted by down-regulation of p53 though iASPP pathway.

Several limitations of the current study merit comment. Neuron necrosis and apoptosis are the key cellular events in neurologic injuries under ischemia-reperfusion. It is not known whether neuroprotective effects against neuron necrosis rather than antiapoptosis effects contribute to the neuroprotection of inhibition of miRNA-124 in spinal cords. The mechanisms by which miRNA-124 regulates mitophagy in spinal cords under ischemia-reperfusion have not investigated fully. Further investigation is needed to identify whether other target proteins of miRNA-124, aside from iASPP, contribute to neuroprotection against spinal cord ischemia. Mitochondrial probes with markers of the autophagic machinery might be used to assess mitophagy more directly.

In conclusion, inhibition of miRNA-124 can provide a powerful neuroprotection on spinal cords against ischemia-reperfusion injury, which may be mediated by both induction of mitophagy and antiapoptotic effects. To our knowledge, this is the first report that mitophagy plays an important role in the neuroprotection against spinal cord ischemia by targeting miRNAs. When a similar rat model is used, overexpression of miRNA- $21^{9}$ and inhibition of miRNA- $320^{10}$ have been indicated to be neuroprotective against spinal cord ischemia. The current study further expanded the understanding of miRNAs as neuroprotective molecular targets against ischemic injury of spinal cords. 


\section{Conflict of Interest Statement}

Authors have nothing to disclose with regard to commercial support.

\section{References}

1. Wong DR, Parenti JL, Green SY, Chowdhary V, Liao JM, Zarda S, et al. Open repair of thoracoabdominal aortic aneurysm in the modern surgical era: contemporary outcomes in 509 patients. J Am Coll Surg. 2011;212:569-79.

2. Dias NV, Sonesson B, Kristmundsson T, Holm H, Resch T. Short-term outcome of spinal cord ischemia after endovascular repair of thoracoabdominal aortic aneurysms. Eur J Vasc Endovasc Surg. 2015;49:403-9.

3. Kim VN, Han J, Siomi MC. Biogenesis of small RNAs in animals. Nat Rev Mol Cell Biol. 2009; 10:126-39.

4. Bak M, Silahtaroglu A, Møller M, Christensen M, Rath MF, Skryabin B, et al. MicroRNA expression in the adult mouse central nervous system. RNA. 2008; $14: 432-44$.

5. Saugstad JA. MicroRNAs as effectors of brain function with roles in ischemia and injury, neuroprotection, and neurodegeneration. J Cereb Blood Flow Metab. 2010;30:1564-76.

6. Dharap A, Bowen K, Place R, Li LC, Vemuganti R. Transient focal ischemia induces extensive temporal changes in rat cerebral microRNAome. J Cereb Blood Flow Metab. 2009;29:675-87.

7. Liu NK, Wang XF, Lu QB, Xu XM. Altered microRNA expression following traumatic spinal cord injury. Exp Neurol. 2009;219:424-9.

8. Wang Y, Gu T, Shi E, Yu L, Wang C, Zhang Y, et al. Inhibition of microRNA-29c protects the brain in a rat model of prolonged hypothermic circulatory arrest. J Thorac Cardiovasc Surg. 2015;150:675-84.

9. He F, Ren Y, Shi E, Liu K, Yan L, Jiang X. Overexpression of microRNA-21 protects spinal cords against transient ischemia. J Thorac Cardiovasc Surg. 2016;152:1602-8.

10. He F, Shi E, Yan L, Li J, Jiang X. Inhibition of micro-ribonucleic acid-320 attenuates neurologic injuries after spinal cord ischemia. J Thorac Cardiovasc Surg. 2015; 150:398-406.

11. Kubli DA, Gustafsson $\AA$ B. Mitochondria and mitophagy: the yin and yang of cell death control. Circ Res. 2012;111:1208-21.

12. Mizushima N, Levine B, Cuervo AM, Klionsky DJ. Autophagy fights disease through cellular selfdigestion. Nature. 2008;451:1069-75.

13. Bin-Umer MA, McLaughlin JE, Butterly MS, McCormick S, Tumer NE. Elimination of damaged mitochondria through mitophagy reduces mitochondrial oxidative stress and increases tolerance to trichothecenes. Proc Natl Acad Sci U S A. 2014;111:11798-803.

14. Li P, Jiao J, Gao G, Prabhakar BS. Control of mitochondrial activity by miRNAs. J Cell Biochem. 2012;113:1104-10.

15. Frankel LB, Wen J, Lees M, Høyer-Hansen M, Farkas T, Krogh A, et al. microRNA-101 is a potent inhibitor of autophagy. EMBO J. 2011;30:4628-41.

16. Huang C, Xiao J, Zhu X, He B, Zhang Y, Kang B, et al. MiR-204 regulates cardiomyocyte autophagy induced by ischemia-reperfusion through LC3-II. J Biomed Sci. 2011;18:35.

17. Zhu H, Wu H, Liu X, Li B, Chen Y, Ren X, et al. Regulation of autophagy by a beclin 1-targeted microRNA, miR-30a, in cancer cells. Autophagy. 2009;5: 816-23.

18. Lagos-Quintana M, Rauhut R, Yalcin A, Meyer J, Lendeckel W, Tuschl T. Identification of tissue-specific microRNAs from mouse. Curr Biol. 2002;12:735-9.

19. Cheng LC, Pastrana E, Tavazoie M, Doetsch F. miR-124 regulates adult neurogenesis in the subventricular zone stem cell niche. Nat Neurosci. 2009;12:399-408.

20. Weng H, Shen C, Hirokawa G, Ji X, Takahashi R, Shimada K, et al. Plasma miR124 as a biomarker for cerebral infarction. Biomed Res. 2011;32:135-41.

21. Zhu F, Liu JL, Li JP, Xiao F, Zhang ZX, Zhang L. MicroRNA-124 (miR-124) regulates Ku70 expression and is correlated with neuronal death induced by ischemia/reperfusion. J Mol Neurosci. 2014;52:148-55.

22. Lewis BP, Shih IH, Jones-Rhoades MW, Bartel DP, Burge CB. Prediction of mammalian microRNA targets. Cell. 2003;115:787-98.
23. Liu X, Li F, Zhao S, Luo Y, Kang J, Zhao H, et al. MicroRNA-124-mediated regulation of inhibitory member of apoptosis-stimulating protein of p53 family in experimental stroke. Stroke. 2013;44:1973-80.

24. Taira Y, Marsala M. Effect of proximal arterial perfusion pressure on function, spinal cord blood flow, and histopathologic changes after increasing intervals of aortic occlusion in the rat. Stroke. 1996;27:1850-8.

25. Di Y, He YL, Zhao T, Huang X, Wu KW, Liu SH, et al. Methylene blue reduces acute cerebral ischemic injury via the induction of mitophagy. Mol Med. 2015; 21:420-9.

26. Tan JR, Koo YX, Kaur P, Liu F, Armugam A, Wong PT, et al. microRNAs in stroke pathogenesis. Curr Mol Med. 2011;11:76-92.

27. Doeppner TR, Doehring M, Bretschneider E, Zechariah A, Kaltwasser B, Müller B, et al. MicroRNA-124 protects against focal cerebral ischemia via mechanisms involving Usp14-dependent REST degradation. Acta Neuropathol. 2013;126:251-65.

28. Li Y, Ahmad A, Sarkar FH. ASPP and iASPP: implication in cancer development and progression. Cell Mol Biol (Noisy-le-grand). 2015;61:2-8.

29. Hong LZ, Zhao XY, Zhang HL. p53-mediated neuronal cell death in ischemic brain injury. Neurosci Bull. 2010;26:232-40.

30. Li J, Chen G, Gao X, Shen C, Zhou P, Wu X, et al. p53 participates in the protective effects of ischemic post-conditioning against OGD-reperfusion injury in primary cultured spinal cord neurons. Neurosci Lett. 2016;638:129-34.

31. Andres AM, Ratliff EP, Hernandez G, Lee P, Gottlieb RA. Preconditioning involves selective mitophagy mediated by Parkin and p62/SQSTM1. PLoS One. 2011;6:e20975.

32. Andres AM, Hernandez G, Lee P, Huang C, Ratliff EP, Sin J, et al. Mitophagy is required for acute cardioprotection by simvastatin. Antioxid Redox Signal. 2014; 21:1960-73.

33. Schapira AH, Olanow CW, Greenamyre JT, Bezard E. Slowing of neurodegeneration in Parkinson's disease and Huntington's disease: future therapeutic perspectives. Lancet. 2014;384:545-55.

34. Santos RX, Correia SC, Carvalho C, Cardoso S, Santos MS, Moreira PI. Mitophagy in neurodegeneration: an opportunity for therapy? Curr Drug Targets. 2011; 12:790-9.

35. Zhang X, Yan H, Yuan Y, Gao J, Shen Z, Cheng Y, et al. Cerebral ischemiareperfusion-induced autophagy protects against neuronal injury by mitochondrial clearance. Autophagy. 2013;9:1321-33.

36. Zhang X, Yuan Y, Jiang L, Zhang J, Gao J, Shen Z, et al. Endoplasmic reticulum stress induced by tunicamycin and thapsigargin protects against transient ischemic brain injury: Involvement of PARK2-dependent mitophagy. Autophagy. 2014;10:1801-13.

37. Niizuma K, Yoshioka H, Chen H, Kim GS, Jung JE, Katsu M, et al. Mitochondrial and apoptotic neuronal death signaling pathways in cerebral ischemia. Biochim Biophys Acta. 2010;1802:92-9.

38. Ji W, Wei S, Hao P, Xing J, Yuan Q, Wang J, et al. Aldehyde dehydrogenase 2 has cardioprotective effects on myocardial ischaemia/reperfusion injury via suppressing mitophagy. Front Pharmacol. 2016;7:101.

39. Yu S, Zheng S, Leng J, Wang S, Zhao T, Liu J. Inhibition of mitochondrial calcium uniporter protects neurocytes from ischemia/reperfusion injury via the inhibition of excessive mitophagy. Neurosci Lett. 2016;628:24-9.

40. Zhang XM, Zhang L, Wang G, Niu W, He Z, Ding L, et al. Suppression of mitochondrial fission in experimental cerebral ischemia: the potential neuroprotective target of p38 MAPK inhibition. Neurochem Int. 2015;90:1-8.

41. Wang DB, Kinoshita C, Kinoshita Y, Morrison RS. p53 and mitochondrial function in neurons. Biochim Biophys Acta. 2014;1842:1186-97.

42. Hoshino A, Matoba S, Iwai-Kanai E, Nakamura H, Kimata M, Nakaoka M, et al. p53-TIGAR axis attenuates mitophagy to exacerbate cardiac damage after ischemia. J Mol Cell Cardiol. 2012;52:175-84.

Key Words: microRNA-124, spinal cord, ischemia-reperfusion, mitophagy, apoptosis 